\title{
Surgery for predominant lesion in nonlocalized bronchiectasis
}

Jie Dai, PhD, Xinsheng Zhu, MD, Dongliang Bian, MD, Ke Fei, MD, Gening Jiang, MD, and

Peng Zhang, MD

\section{ABSTRACT}

Objective: Patients with nonlocalized bronchiectasis are encountered commonly; however, there is little information regarding surgical intervention in this patient population. The aim of this study was to evaluate symptomatic response and safety of anatomic resection of the predominant lesion via the use of lobectomy for the management of nonlocalized bronchiectasis.

Methods: We reviewed the medical records of 37 consecutive patients who underwent lobectomy via thoracotomy for nonlocalized bronchiectasis between 2010 and 2013. The main surgical indications were nonlocalized bronchiectasis with one predominant lesion, failure of medical treatment, and adequate cardiopulmonary reserve. The predominant lesion was determined by preoperative computed tomography and/or bronchoscopy. Preoperative symptoms were compared with postoperative symptoms and analyzed by the use of paired techniques.

Results: The mean patient age was $54.5 \pm 6.4$ years. There was no operative mortality. Postoperative complications occurred in $8(21.6 \%)$ patients, including 1 with empyema, 1 with persistent air leak, and 6 with minor transient complications, all of which were manageable without any reoperation. After lobectomy, the median extent of residual bronchiectatic areas in the remaining lungs was $25 \%$ (range, $12.5 \%-42.9 \%)$. The frequency of acute infection $(5.3 \pm 2.1$ year vs $1.8 \pm 2.3 /$ year $)$ and hemoptysis $(4.9 \pm 2.8 /$ year vs $1.1 \pm 0.7 /$ year $)$ decreased significantly and the amount of sputum also decreased $(37.1 \pm 3.4 \mathrm{~mL} /$ day vs $10.7 \pm 4.6 \mathrm{~mL} / \mathrm{day})$. Twenty-three $(62.2 \%)$ patients were asymptomatic after surgery, $10(27.0 \%)$ were symptomatic with clinical improvement, and $4(10.8 \%)$ had no change or worsened.

Conclusions: Lobectomy for the predominant lesion is a safe procedure in the surgical treatment of nonlocalized bronchiectasis and leads to significant relief of symptoms with good rates of satisfaction. (J Thorac Cardiovasc Surg 2017;153:979-85)

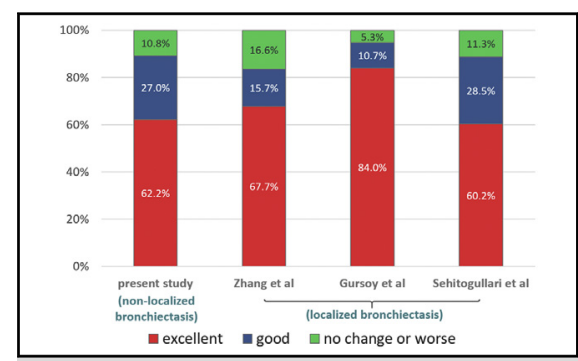

Patient satisfaction after surgery.

\section{Central Message}

Lobectomy for the predominant lesion in nonlocalized bronchiectasis can improve symptoms significantly with comparable satisfaction rates with localized bronchiectasis.

\section{Perspective}

Nonlocalized bronchiectasis is common. This study investigates the safety of lobectomy for predominant lesion in nonlocalized bronchiectasis and finds no operative mortality and an acceptable morbidity rate. Patients experience significant symptom improvement, and most are satisfied with the outcome. Lobectomy can be considered in selected patients whose symptoms are not controlled by medical therapy.

See Editorial Commentary page 986.
Bronchiectasis is defined as an abnormal and irreversible dilatation of the bronchi, often associated with chronic productive cough, airway obstruction, and recurrent

\footnotetext{
From the Department of Thoracic Surgery, Shanghai Pulmonary Hospital Tongji University, Shanghai, People's Republic of China.

Supported by National Natural Science Foundation of China (NSFC 81200034 and 81470244).

Read at the 24th European Conference on General Thoracic Surgery, Naples, Italy, May 29 to June 1, 2016.

Received for publication June 16, 2016; revisions received Nov 18, 2016; accepted for publication Dec 6, 2016; available ahead of print Jan 7, 2017.

Address for reprints: Peng Zhang, MD, Department of Thoracic Surgery, Shanghai Pulmonary Hospital Tongji University, No. 507 Zhengmin Rd, Shanghai 200433,

People's Republic of China (E-mail: zhangpeng1121@aliyun.com). 0022-5223/\$36.00

Copyright $(2016$ by The American Association for Thoracic Surgery http://dx.doi.org/10.1016/j.jtcvs.2016.12.004
}

infections. ${ }^{1}$ Although the prevalence of bronchiectasis is not well characterized (from 3.7 to 52 per 100,000 adults ${ }^{2}$ ), it has become a major medical concern in developing countries, such as China. ${ }^{3-5}$

The role of surgical intervention in localized bronchiectasis has been well established, ${ }^{2,6}$ and indications for surgery include failure of medical treatment, hemoptysis, and progressive disease with recurrent infections.

Scanning this QR code will take you to supplemental figure and video for this article. 


\section{Abbreviation and Acronym \\ $\mathrm{CT}=$ computed tomography}

Complete resection is pivotal in preventing postoperative infectious complications and disease recurrence. ${ }^{8,9}$ Therefore, surgery has not been considered traditionally for nonlocalized bronchiectasis.

In patients with nonlocalized bronchiectasis in whom symptoms are not controlled by conservative medical therapy, however, the role of surgical resection for the predominant lesion should be re-examined. In our center, 37 patients with nonlocalized bronchiectasis received this therapeutic option. Surgery was indicated for patients with nonlocalized bronchiectasis provided that (1) patient symptoms persisted despite appropriate medical treatment, (2) the most affected area (ie, the predominant lesion) could be identified, and (3) cardiopulmonary function was compatible with the anesthetic risk. In this study, we aimed to evaluate symptomatic response and safety of anatomic resection of the predominant lesion by using lobectomy for the management of nonlocalized bronchiectasis.

\section{MATERIALS AND METHODS \\ Study Population}

This study was approved by the review board of Tongji University. Written informed consent was obtained from all patients. Nonlocalized bronchiectasis was defined as the bronchiectatic areas involving multiple segments and lobes. ${ }^{10}$ Patients who had bronchiectasis with cystic fibrosis, bronchiectasis combined with aspergilloma, and surgery for emergency reasons were excluded from this study. We reviewed the medical records of $37 \mathrm{pa}-$ tients with nonlocalized bronchiectasis who underwent lobectomy for the predominant lesion in our department between January 2010 and December 2013.

The initial diagnosis of bronchiectasis was based on detailed patient medical records and documented bronchial wall dilatation on thoracic computed tomography (CT). All patients received standard medical treatment before operation, including systemic antibiotics, nonirritant expectorants, and anti-inflammatory agents according to our previous experience. ${ }^{4}$ Failure of medical treatment was defined as frequent exacerbation interfering with normal professional or social life or requiring multiple hospitalizations. ${ }^{4}$ The predominant lesion was located in the most severely affected area on CT scan (Figure 1), or in the area involved in hemoptysis through bronchoscopic examination. Surgical resection was not considered for patients with diffuse bronchiectasis. A predicted forced expiratory volume in 1 second of greater than $60 \%$ was considered adequate for surgery.

\section{Preoperative Evaluation}

Details of patient preoperative evaluation have been reported in previous publications. ${ }^{3,4,11}$ To summarize, thoracic CT was performed to locate the predominant lesion. The extent of bronchiectatic lesions that was expected to be left behind was expressed as a proportion of the remaining lungs, which was calculated by the anatomic method, where the total number of segments for both lungs was deemed 19 (10 in the right lung and 9 in the left lung). ${ }^{12}$ Bronchoscopy was used for the assessment of endobronchial membrane (excluding proximal obstruction), characterization of mycobacterial colonization, and for removal of secretions. The side opposite the predominant lesion should be examined first to minimize any potential contamination. For patients with compromised pulmonary function, quantitative pulmonary ventilation and perfusion scan was performed to estimate the postoperative lung function for operative consideration. Empiric antibiotic treatment was given with cephalosporin for a minimum of 48 hours before the operation if patients were not receiving antibiotic treatment. For those who were taking antibiotics, the regimen was continued in the perioperative setting. Patients were instructed by our nurses to collect their daily sputum in a given cup and to estimate the volume visually. They were prepared strictly at least 2 weeks until the daily sputum volume was less than $20 \mathrm{~mL}$. For patients who failed to reach this threshold, a repeat sputum culture was performed, and antibiotics were modified subsequently on the basis of antibiotic sensitivity results. ${ }^{2}$ Combination antibiotics were administrated if required. Additional medical treatment, such as anti-inflammatory medication and chest physiology therapy, was provided by a multidisciplinary team involving pulmonary and infectious disease consultants.

\section{Surgical Technique}

Posterolateral thoracotomy was performed in all patients. A doublelumen endotracheal tube was used to avoid the intraoperative contamination of the opposite side of the lung. Lobectomy was performed to remove the predominant lesion, including right upper lobectomy in 7 patients, right middle lobectomy in 9, right lower lobectomy in 5, left upper lobectomy in 6 , and left lower lobectomy in 10 . The minor lesions, which resided ipsilaterally in 2 patients and contralaterally in 35, were left untouched during the operation (Figure 2). Excessive bronchial dissection was avoided, and peribronchial tissues were preserved. Extrapleural dissection was performed to avoid spillage of lung content into pleural space. The order in the treatment of the hilum of lung was from pulmonary artery to pulmonary vein and then to the bronchus. Distorted bronchial arteries were isolated carefully and ligated or severed by ultrasonic device. The bronchial stump was closed with a mechanical stapler and reinforced with an intercostal muscle flap in 16 cases and a pedicled parietal pleural flap in 21 cases (Figure E1). After lobectomy, careful hemostasis was performed, and, if any early signs of
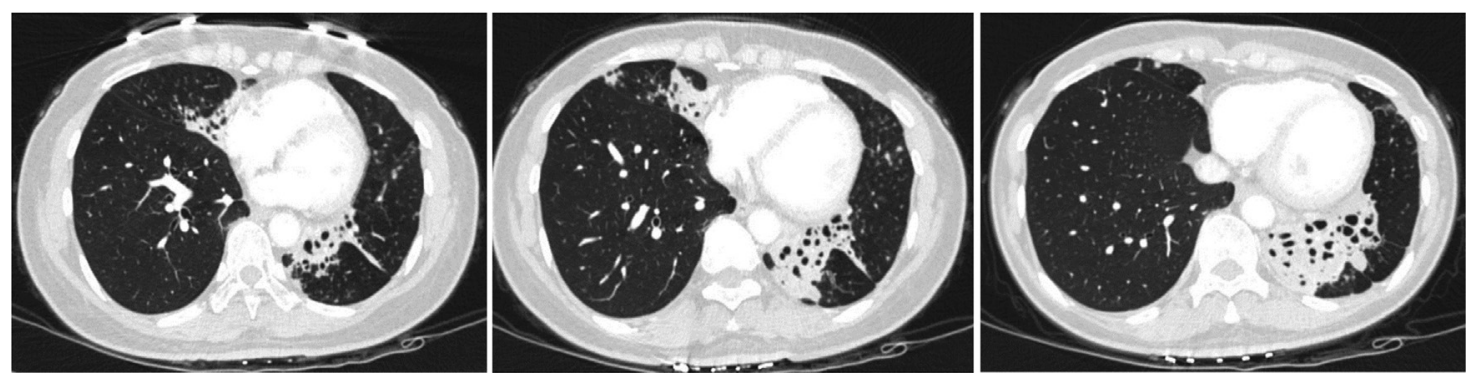

FIGURE 1. A 55-year old male patient suffered from bilateral bronchiectasis. The predominant lesion in the left lower lobe, the minor one in the right middle lobe, and some scattered lesions in both sides. 


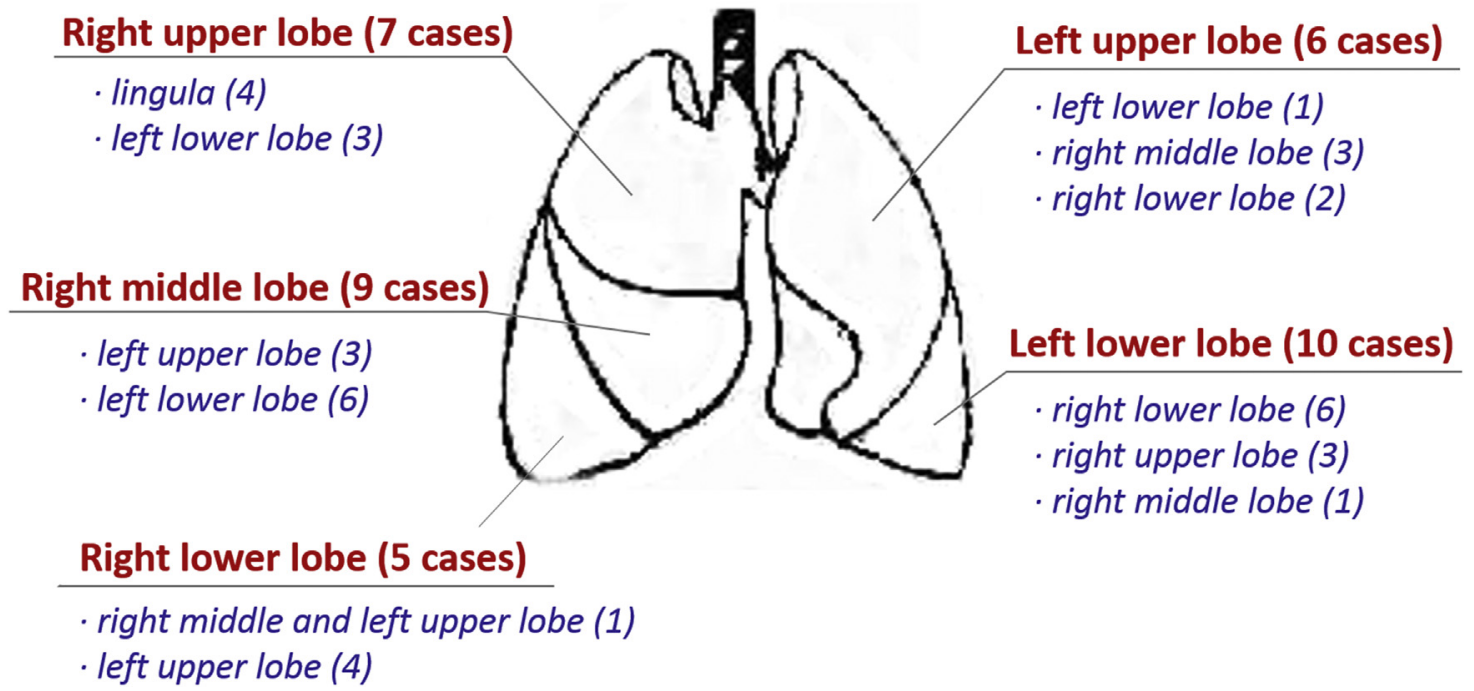

FIGURE 2. Distribution of involved area in nonlocalized bronchiectasis. The predominant lesions (in red) were resected by lobectomy and the minor lesions (in blue) were left untouched. The numbers in parentheses represent the number of lesion.

pleural infection were present, pleural space irrigation was conducted with $0.5 \%$ neomycin $(500 \mathrm{mg} / \mathrm{L})$. Two chest drains were placed at the site of bronchial stump. The bronchial suture was checked bronchoscopically, and any secretion was removed from the airways. All specimens had pathologic confirmation of bronchiectasis.

\section{Postoperative Treatment and Assessment}

Intensive postoperative care was conducted by a specialized team that included chest physicians, thoracic surgeons, and well-trained nurses. Systematic antibiotic treatment was continued for 5 days after surgery, or longer if the patient had inflammatory symptoms. Active cough and early ambulation were encouraged on postoperative day 1 to facilitate expectoration and promote lung re-expansion. ${ }^{4}$ Percussive chest physiotherapy was performed 3 to 4 times daily. Chest tubes were removed if (1) the patient's vital status was stable and routine blood tests were in the normal range, (2) the amount of drainage diminished to 80 to $100 \mathrm{~mL}$ or less per day and the color was clear, and (3) there was no evidence of air leak and lung re-expansion was confirmed by a chest radiograph. Bronchoscopic sputum aspiration was used if patients had postoperative atelectasis, and thoracentesis was required if significant pleural effusion was present after removal of the chest tubes. Postoperative complications were defined as those occurred during hospitalization or within 30 days of treatment. Persistent air leak was defined as air leak that lasts more than 2 weeks. ${ }^{4,13}$

Follow-up was completed in all patients with a mean period of 15.3 months (range, 11.9-20.4 months). The outcome of surgery was evaluated at 1 year postoperatively with a structured questionnaire from our previous study. ${ }^{3}$ Patients were assessed for the frequency of acute infection (n/year) and hemoptysis (n/year) in the following 1 year, and for the dyspnea score of Fletcher and colleagues ${ }^{14}$ and daily sputum volume (mL/ day) at the time of follow-up appointment. Patient satisfaction with regard to symptom relief was evaluated according to the following criteria ${ }^{13}$ : excellent (absence of preoperative symptoms), good (alleviation in preoperative symptoms), no change (no reduction in preoperative symptoms), and worse (frequent exacerbation of disease requiring hospitalization).

\section{Statistical Analysis}

Clinical data were summarized with the mean and standard deviation or median and interquartile range as appropriate based on normality of distribution of continuous data. Categorical variables were summarized with proportions. The preoperative and postoperative patient outcomes were compared with a paired $t$ test for continuous variables and the McNemar test for categorical variables. Independent factors for poor prognosis were studies by logistic regression analyses. A $P$ value less than .05 was considered statistically significant. The outcomes of patient satisfaction as to symptom relief in nonlocalized bronchiectasis were compared with those in localized bronchiectasis reported in the previous studies ${ }^{4,15,16}$ using stacked bar graph. All statistical analyses were performed by SPSS Statistics 19 (IBM Corp, Armonk, NY).

\section{RESULTS}

Patient clinical characteristics are listed in Table 1. There were 10 male and 27 female patients, with a mean age of $54.5 \pm 6.4$ years. All patients had chronic symptoms for a mean period of $62.8 \pm 8.2$ months. Bacterial infection was preoperatively proven in all patients.

The mean operative time was $174 \pm 82$ minutes, and the mean intraoperative blood loss was $215 \pm 60 \mathrm{~mL}$. No perioperative blood transfusion was needed. After surgery, the median extent of residual bronchiectatic areas in the remaining lungs was $25 \%$ (range, $12.5 \%-42.9 \%$ ). There was no perioperative mortality. Postoperative complications occurred in $8(21.6 \%)$ patients, including empyema $(\mathrm{n}=1)$, which was treated with chest tube drainage, persistent air leak $(\mathrm{n}=1)$, postoperative pneumonia $(\mathrm{n}=2)$, atelectasis requiring bronchoscopy $(\mathrm{n}=2)$, and cardiac arrhythmia $(\mathrm{n}=2)$. The mean duration of hospital stay was $13.0 \pm 6.5$ days.

Patients experienced marked symptomatic improvement after 1 year postoperatively (Table 2). The postoperative frequency of acute infection and hemoptysis decreased significantly (both $P<.01$ ). The daily volume of sputum was reduced by $26.3 \pm 6.1 \mathrm{~mL}(P<.01)$. Postoperative sputum culture studies became sterile in $13(35.1 \%)$ of 37 initially infected patients $(P<.01)$. Evaluation of patient satisfaction revealed that $23(62.2 \%)$ patients were 
TABLE 1. Characteristics of study population $(\mathrm{N}=37)$

\begin{tabular}{lc}
\hline \multicolumn{1}{c}{ Number $(\%)$ or mean \pm SD } \\
\hline Sex & \\
$\quad$ Male & $10(27.0)$ \\
Female & $27(73.0)$ \\
Age, y & $54.5 \pm 6.4$ \\
Smoking history & \\
$\quad$ Never & $19(51.4)$ \\
Former & $13(35.1)$ \\
Current & $5(13.5)$ \\
Body mass index, kg/m ${ }^{2}$ & $21.7 \pm 3.6$ \\
FEV1 \% predicted, mean & $75.2 \pm 16.9$ \\
Comorbid diseases & \\
Chronic obstructive pulmonary disease & $11(29.7)$ \\
Hypertension & $6(16.2)$ \\
Chronic renal insufficiency & $2(5.4)$ \\
Presenting symptom & \\
Cough & $37(100)$ \\
Hemoptysis & $11(29.7)$ \\
Purulent sputum & $16(43.2)$ \\
\hline
\end{tabular}

FEVI, Forced expiratory volume in 1 second.

"excellent" after surgery, $10(27.0 \%)$ were "good," and only $4(10.8 \%)$ were "no change or worse" (Figure 3, A). Among the 4 patients with no postoperative improvement, 3 had concomitant chronic obstructive pulmonary disease. Two patients underwent upper lobectomy, 1 middle lobectomy, and 1 lower lobectomy. The extent of residual bronchiectatic areas in these 4 patients was $42.8 \%, 35.7 \%$, $33.3 \%$, and $12.5 \%$, respectively. No significant predictors for poor prognosis were identified.

\section{DISCUSSION}

Bronchiectasis is a pathologic endpoint that results from many disease processes and poses an increasing burden on healthcare systems worldwide. ${ }^{17,18}$ Patients with nonlocalized bronchiectasis currently are being encountered in clinical practice,,${ }^{5,7}$ in whom the difficulty in relieving symptoms through conservative therapy leads to the consideration of surgery for the most affected area. We aimed to assess the safety and efficacy of this

TABLE 2. Outcomes after surgery for predominant lesion in nonlocalized bronchiectasis $(N=37)$

\begin{tabular}{lccc}
\hline \multicolumn{1}{c}{ Outcome } & Preoperative & Postoperative & $\boldsymbol{P}$ value \\
\hline $\begin{array}{l}\text { Frequency of acute } \\
\text { infection, n/y }\end{array}$ & $5.3 \pm 2.1$ & $1.8 \pm 2.3$ & $2.82 \times 10^{-13}$ \\
$\begin{array}{l}\text { Frequency of } \\
\text { hemoptysis, n/y }\end{array}$ & $4.9 \pm 2.8$ & $1.1 \pm 0.7$ & $1.25 \times 10^{-16}$ \\
$\begin{array}{l}\text { Fletcher dyspnea score } \\
\begin{array}{l}\text { Volume of sputum, } \\
\text { mL/d }\end{array}\end{array}$ & $1.2 \pm 0.9$ & $0.9 \pm 0.7$ & .003 \\
$\quad$ Sputum culture/positive & $37(100 \%)$ & $13(35.1 \%)$ & $1.19 \times 10^{-7}$ \\
\hline
\end{tabular}

Values are mean \pm SD or $\mathrm{n}(\%)$.

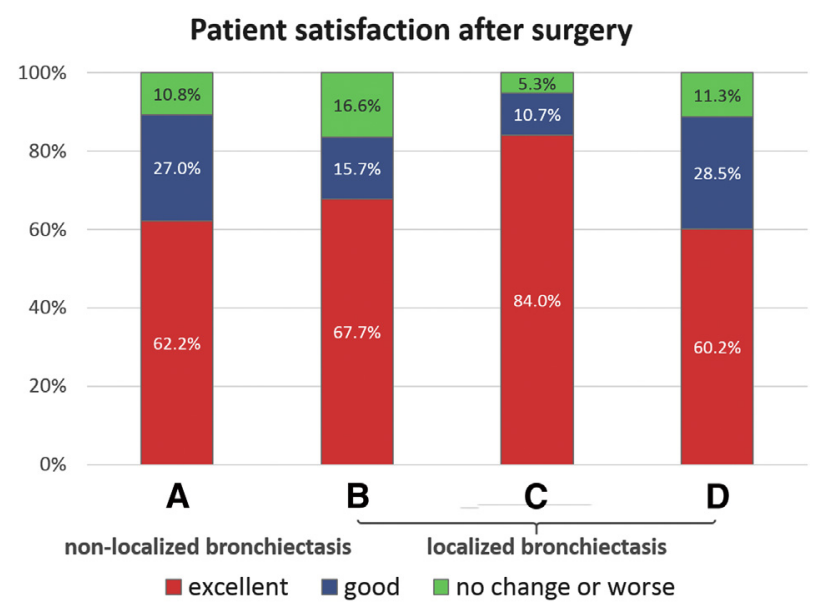

FIGURE 3. Comparison of patient satisfaction with regard to symptom relief after surgery between non-localized and localized bronchiectasis. A, Data from the present study; B, data from the study by Zhang and colleagues ${ }^{4} ; \mathrm{C}$, data from the study by Gursoy and colleagues ${ }^{15}$; and D, data from the study by Sehitogullari and colleagues. ${ }^{16}$

therapeutic option for nonlocalized bronchiectasis, and our findings demonstrated that lobectomy can be performed safely with an acceptable postoperative morbidity rate and patient symptoms can be improved significantly with good satisfaction rates after resection of the predominant lesion in the lung.

Surgery has been used for bronchiectasis with the principal aim of improving symptoms as well as quality of life. ${ }^{6,15,19}$ A recent meta-analysis pooling 35 studies confirmed the efficacy of surgical intervention in patients with noncystic fibrosis bronchiectasis ${ }^{20}$; however, operations in these previous studies were performed mainly in the setting of localized bronchiectasis. Surgery in nonlocalized bronchiectatic disease was reserved only for certain life-threatening symptoms (ie, massive hemoptysis) and infection-related complications (eg, pulmonary abscess). ${ }^{2,6}$ In 2003, Mazieres and colleagues ${ }^{21}$ initially evaluated the utility of limited resection in 16 patients with multisegmental bilateral bronchiectasis and showed symptomatic relief after operation. Schneiter and colleagues ${ }^{10}$ subsequently conducted a comparative study and found a similar postoperative patient satisfaction between those with localized and those with nonlocalized bronchiectasis, although these comparisons were not performed through a strict statistical analysis.

On the basis of these previous studies, we set forth to investigate the feasibility of lobectomy for predominant lesion in nonlocalized bronchiectasis while minor diseased areas were left behind in the remaining lungs. The perioperative results showed no operative mortality and an acceptable morbidity rate of $21.6 \%$, which was comparable with those in localized bronchiectasis in other series (Table 3). To note, 2 major complications, empyema and persistent air leak, occurred but 
TABLE 3. Perioperative outcomes of surgery for bronchiectasis from recent studies

\begin{tabular}{|c|c|c|c|c|}
\hline Author (year of publication) & Type of bronchiectasis & No. of patients & Mortality, n (\%) & Morbidity, n (\%) \\
\hline Present study & Nonlocalized & 37 & 0 & $8(21.6)$ \\
\hline Balci and colleagues $(2014)^{6}$ & Localized & 86 & $1(1.1)$ & $14(14.6)$ \\
\hline Al-Refaie and colleagues $(2013)^{23}$ & Localized & 138 & 0 & $18(13)$ \\
\hline Sehitogullari and colleagues $(2011)^{16}$ & Localized & 129 & $1(0.7)$ & $29(22.4)$ \\
\hline Zhang and colleagues $(2010)^{4}$ & Localized & 790 & $9(1.1)$ & $128(16.2)$ \\
\hline Gursoy and colleagues $(2010)^{15}$ & Localized & 92 & $1(1)$ & $15(16)$ \\
\hline Eren and colleagues $(2007)^{13}$ & Localized & 143 & $2(1.3)$ & $33(23.0)$ \\
\hline Schneiter and colleagues $(2005)^{10}$ & Combined & 48 & 0 & $6(12.5)$ \\
\hline
\end{tabular}

both were manageable without the need for reoperation. Postoperative empyema was common in bronchiectasis, ${ }^{6,16}$ and most could be treated successfully by tube thoracostomy. In addition, persistent air leak was frequently noted after operation, with a reported rate of $4.8 \%$ to $6.1 \% .^{13,22}$ Thus, extreme caution should be taken during the dissection of lung parenchyma.

On the basis of our previous experience in management of bronchiectasis, ${ }^{3,4}$ we have established certain important care protocols to reduce the risk of postoperative

non-localized bronchiectasis: persistent

symptoms despite medical treatment

- To identify the predominant lesion via CT or bronchoscopy - To assess anesthetic risk via cardiopulmonary tests

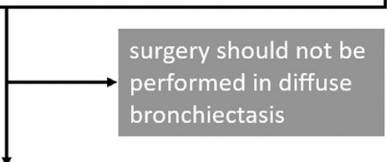

Preope a) sputum culture and daily sputum volume collection

rative b) antibiotics: empirical (cephalosporin) or based on

manag previous bacteriology results

c) airway preparation: atomization, postural drainage,

ement d) bronchoscopy: assessment for endobronchial membran

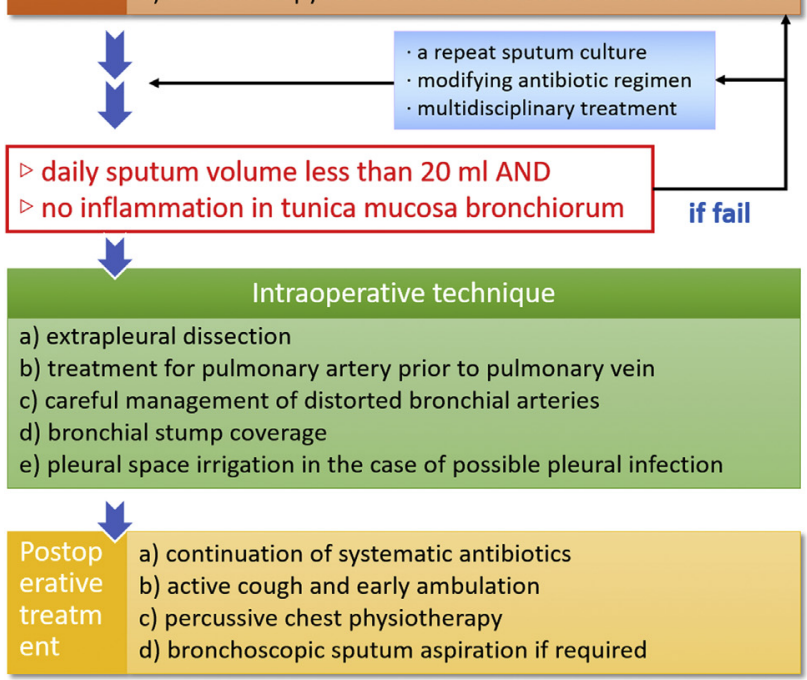

FIGURE 4. Algorithm for perioperative management of patients with nonlocalized bronchiectasis. complications (Figure 4). Preoperatively, patients must be prepared strictly until sputum is less than $20 \mathrm{~mL} /$ day with little purulence and bronchoscopy shows no engorgement or edema in the tunica mucosa bronchiorum. Eren and colleagues ${ }^{13}$ found patients in whom bronchoscopy was not performed had a significantly greater rate of postoperative complications. Although preoperative bronchoscopic examination is not necessary for a diagnosis of bronchiectasis, it serves as an important adjuvant method, in the setting of nonlocalized bronchiectasis, to locate dominant lesion, exclude gastric aspiration, evaluate inflammatory condition, and to perform bronchial toilet. ${ }^{2,21}$ Meanwhile, the control of sputum volume is critically important. Jin and colleagues ${ }^{11}$ revealed that daily sputum volume $(>30 \mathrm{~mL})$ was associated with the worse surgical outcome. Therefore, surgery should not be performed in cases of severe inflammation until adequate control has been achieved. Pleural adhesion is commonly encountered during the operation, ${ }^{6}$ so careful extrapleural dissection is suggested to reduce the potential risk of postoperative infectious and hemorrhagic complications. The pulmonary artery should be treated before the pulmonary vein to avoid pulmonary congestion. In addition, because of a high incidence of postoperative bronchopleural fistula, ${ }^{19}$ bronchial stump coverage is therefore recommended. After a careful surgical planning, we believe that lobectomy can be performed safely with acceptable operative morbidity and mortality rates. Still, patients should be counseled properly about the potential risks, and the operation should be undertaken in experience centers with experienced surgeons.

Patients with bronchiectasis typically presented with signs of recurrent infections, productive cough, and hemoptysis. $^{11,23}$ Our study showed significant symptomatic improvements after lobectomy for the predominant lesion in nonlocalized bronchiectasis. The frequency of acute infection and hemoptysis declined significantly and the amount of sputum decreased. More than $89 \%$ of patients benefited from the operation, which also was comparable with the previously reported satisfaction rates in localized bronchiectasis (Figure 3).,15,16 Surgery could help prevent infective exacerbations and retard disease progression before lungs become grossly diseased. ${ }^{13}$ The 


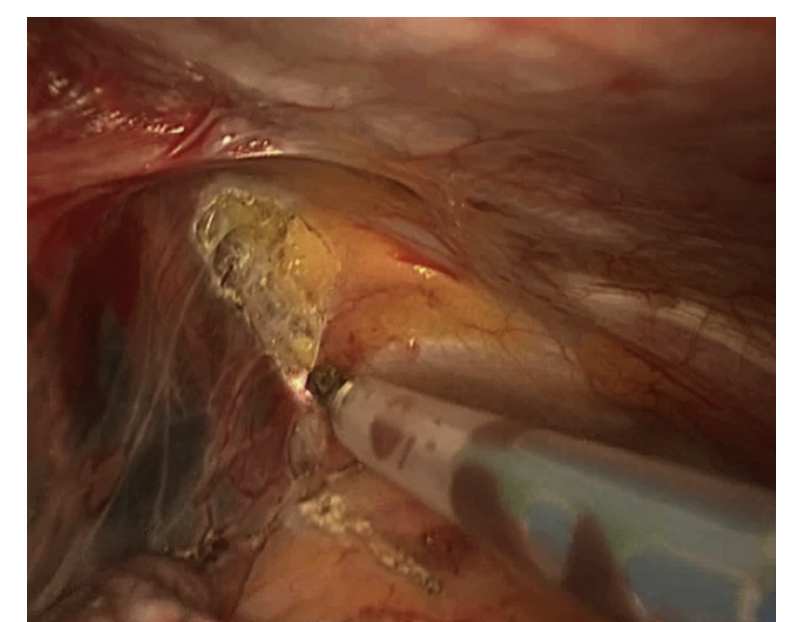

VIDEO 1. Right upper lobectomy for bronchiectasis. Video available at http://www.jtcvsonline.org/article/S0022-5223(16)31667-1/addons.

removal of the predominant lesion may protect the relatively less-affected bronchi from further infectious contamination and rescue the healthy segments. Therefore, primary resection should be considered for predominant lesion in nonlocalized bronchiectatic disease as well. The predominant lesion could be determined reliably on preoperative CT scan because the degree of bronchiectatic radiological abnormality was associated commonly with clinical manifestation and the patient's quality of life. ${ }^{24-27}$ Therefore, a marked symptomatic improvement and a good satisfactory outcome could be obtained in nonlocalized bronchiectatic patients after removal of the predominant lesion, as shown in our study.

It is worth mentioning that the surgical benefit is limited to patients who have only one predominant area of bronchiectatic disease that can be localized by CT, instead of those with diffuse bronchiectasis (ie, cystic fibrosis). The ideal surgical candidate has a heterogeneous distribution of diseased areas. Some areas are damaged severely, whereas others are less affected. In addition, complete resection remains the best cure for bronchiectasis. ${ }^{2}$ For partial resection for nonlocalized bronchiectasis, the basic principle of the operation is to resect as little lung as one can (eg, lobectomy and segmentectomy, rather than pneumonectomy) in a palliative manner. ${ }^{28}$ All patients had less than $50 \%$ bronchiectatic lung after lobectomy, which implies that at least half healthy lung should remain in nonlocalized bronchiectasis when surgery is considered. This should be interpreted with caution, however, because of the small number of patients included in our study. Further validation from independent cohorts is warranted.

This study has a number of limitations. First, it was a retrospective analysis with a relatively small number of patients. The inability to identify predictors for poor prognosis may be attributed to this small sample size. Second, the etiologies of bronchiectasis were not available, which might impact the response to surgical resection. Finally, patients who received minimally invasive pulmonary resection were not included in the study due to inadequate data provided. Further investigations are needed to clarify the role of minimally invasive surgery in this setting (Video 1).

In conclusion, nonlocalized bronchiectasis should not be regarded as a general contraindication to surgery. Our study has demonstrated that lobectomy can be performed safely in patients with nonlocalized bronchiectasis, and patient symptoms can be improved significantly with good satisfaction rates after resection of the predominant lesion in the lung.

\section{Conflicts of Interest Statement}

Authors have nothing to disclose with regard to commercial support.

The authors thank Nan Guo for his valuable assistance in creating Figure E1. We also thank Dr Yuming Zhu for recording the video.

\section{References}

1. Balkanli K, Genc O, Dakak M, Gurkok S, Gozubuyuk A, Caylak H, et al. Surgical management of bronchiectasis: analysis and short-term results in 238 patients. Eur J Cardiothorac Surg. 2003;24:699-702.

2. Pasteur MC, Bilton D, Hill AT. British Thoracic Society guideline for non-CF bronchiectasis. Thorax. 2010;65(suppl 1):11-58.

3. Zhang P, Zhang F, Jiang S, Jiang G, Zhou X, Ding J, et al. Video-assisted thoracic surgery for bronchiectasis. Ann Thorac Surg. 2011;91:239-43.

4. Zhang P, Jiang G, Ding J, Zhou X, Gao W. Surgical treatment of bronchiectasis: a retrospective analysis of 790 patients. Ann Thorac Surg. 2010;90:246-50.

5. Lin JL, Xu JF, Qu JM. Bronchiectasis in China. Ann Am Thorac Soc. 2016;13: 609-16.

6. Balci AE, Balci TA, Ozyurtan MO. Current surgical therapy for bronchiectasis: surgical results and predictive factors in 86 patients. Ann Thorac Surg. 2014;97:211-7.

7. Neves PC, Guerra M, Ponce P, Miranda J, Vouga L. Non-cystic fibrosis bronchiectasis. Interact Cardiovasc Thorac Surg. 2011;13:619-25.

8. Haciibrahimoglu G, Fazlioglu M, Olcmen A, Gurses A, Bedirhan MA. Surgical management of childhood bronchiectasis due to infectious disease. J Thorac Cardiovasc Surg. 2004;127:1361-5.

9. Kutlay H, Cangir AK, Enon S, Sahin E, Akal M, Gungor A, et al. Surgical treatment in bronchiectasis: analysis of 166 patients. Eur J Cardiothorac Surg. 2002; 21:634-7.

10. Schneiter D, Meyer N, Lardinois D, Korom S, Kestenholz P, Weder W. Surgery for non-localized bronchiectasis. Br J Surg. 2005;92:836-9.

11. Jin YX, Zhang Y, Duan L, Yang Y, Jiang GN, Ding JA. Surgical treatment of bronchiectasis: a retrospective observational study of 260 patients. Int J Surg. 2014;12:1050-4.

12. Brunelli A, Kim AW, Berger KI, Addrizzo-Harris DJ. Physiologic evaluation of the patient with lung cancer being considered for resectional surgery: diagnosis and management of lung cancer, 3rd ed: American College of Chest Physicians evidence-based clinical practice guidelines. Chest. 2013;143(5 suppl): e166S-90S.

13. Eren S, Esme H, Avci A. Risk factors affecting outcome and morbidity in the surgical management of bronchiectasis. J Thorac Cardiovasc Surg. 2007; 134:392-8.

14. Fletcher CM, Elmes PC, Fairbairn AS, Wood CH. The significance of respiratory symptoms and the diagnosis of chronic bronchitis in a working population. $\mathrm{Br}$ Med J. 1959;2:257-66.

15. Gursoy S, Ozturk AA, Ucvet A, Erbaycu AE. Surgical management of bronchiectasis: the indications and outcomes. Surg Today. 2010;40:26-30. 
16. Sehitogullari A, Bilici S, Sayir F, Cobanoglu U, Kahraman A. A long-term study assessing the factors influencing survival and morbidity in the surgical management of bronchiectasis. J Cardiothorac Surg. 2011;6:161.

17. Seitz AE, Olivier KN, Steiner CA, Montes DOR, Holland SM, Prevots DR. Trends and burden of bronchiectasis-associated hospitalizations in the United States, 1993-2006. Chest. 2010;138:944-9.

18. Goeminne PC, Nawrot TS, Ruttens D, Seys S, Dupont LJ. Mortality in non-cystic fibrosis bronchiectasis: a prospective cohort analysis. Respir Med. 2014;108: 287-96.

19. Vallilo CC, Terra RM, de Albuquerque AL, Suesada MM, Mariani AW, Salge JM, et al. Lung resection improves the quality of life of patients with symptomatic bronchiectasis. Ann Thorac Surg. 2014;98:1034-41.

20. Fan LC, Liang S, Lu HW, Fei K, Xu JF. Efficiency and safety of surgical intervention to patients with non-cystic fibrosis bronchiectasis: a meta-analysis. Sci Rep. 2015;5:17382.

21. Mazieres J, Murris M, Didier A, Giron J, Dahan M, Berjaud J, et al. Limited operation for severe multisegmental bilateral bronchiectasis. Ann Thorac Surg. 2003; 75:382-7.

22. Hiramatsu M, Shiraishi Y, Nakajima Y, Miyaoka E, Katsuragi N, Kita H, et al. Risk factors that affect the surgical outcome in the management of focal bronchiectasis in a developed country. Ann Thorac Surg. 2012;93:245-50.
23. Al-Refaie RE, Amer S, El-Shabrawy M. Surgical treatment of bronchiectasis: a retrospective observational study of 138 patients. J Thorac Dis 2013;5:228-33.

24. Ooi GC, Khong PL, Chan-Yeung M, Ho JC, Chan PK, Lee JC, et al. High-resolution CT quantification of bronchiectasis: clinical and functional correlation. Radiology. 2002;225:663-72.

25. Lynch DA, Newell J, Hale V, Dyer D, Corkery K, Fox NL, et al. Correlation of CT findings with clinical evaluations in 261 patients with symptomatic bronchiectasis. AJR Am J Roentgenol. 1999;173:53-8.

26. Cobanoglu U, Yalcinkaya I, Er M, Isik AF, Sayir F, Mergan D. Surgery for bronchiectasis: The effect of morphological types to prognosis. Ann Thorac Med. 2011;6:25-32.

27. Eshed I, Minski I, Katz R, Jones PW, Priel IE. Bronchiectasis: correlation of high-resolution CT findings with health-related quality of life. Clin Radiol. 2007;62:152-9.

28. Al-Kattan KM, Essa MA, Hajjar WM, Ashour MH, Saleh WN, Rafay MA. Surgical results for bronchiectasis based on hemodynamic (functional and morphologic) classification. J Thorac Cardiovasc Surg. 2005;130:1385-90.

Key Words: bronchiectasis, surgery, nonlocalized

Readers who found these articles interesting may also like to read the following papers found in recent and future issues of our sister publications, Seminars in Thoracic and Cardiovascular Surgery and Operative Techniques in Thoracic and Cardiovascular Surgery!

\section{Thoracic: Lung}

The Use of Robotic-Assisted Thoracic Surgery for Lung Resection: A Comprehensive Systematic Review John Agzarian. Semin Thoracic Surg 2016: 182-192.

Robotic Assisted Thoracic Surgery Lobectomy versus Video Assisted Thoracic Surgery Lobectomy: Is a Randomized Trial Really Necessary? Robert J. Korst. Semin Thoracic Surg 2016: 193-194.

Estimating the Annual Incremental Cost of Several Complications Following Pulmonary Lobectomy Oliver K. Jawitz. Semin Thoracic Surg 2016: 531-540.

Indocyanine Green Fluorescence Intraoperative Imaging for Hepatic Hydrothorax With a Small Diaphragmatic Defect Naoya Kawakita. Semin Thoracic Surg 2016: 606-608.

A Light in the Darkness: Lighthouse or Anglerfish? Jules Lin. Semin Thoracic Surg 2016: In press.

Chylothorax: Surgical Ligation of the Thoracic Duct Through Thoracotomy Robert A. Meguid. Oper Tech Thorac Cardiovasc 2016: In press. 


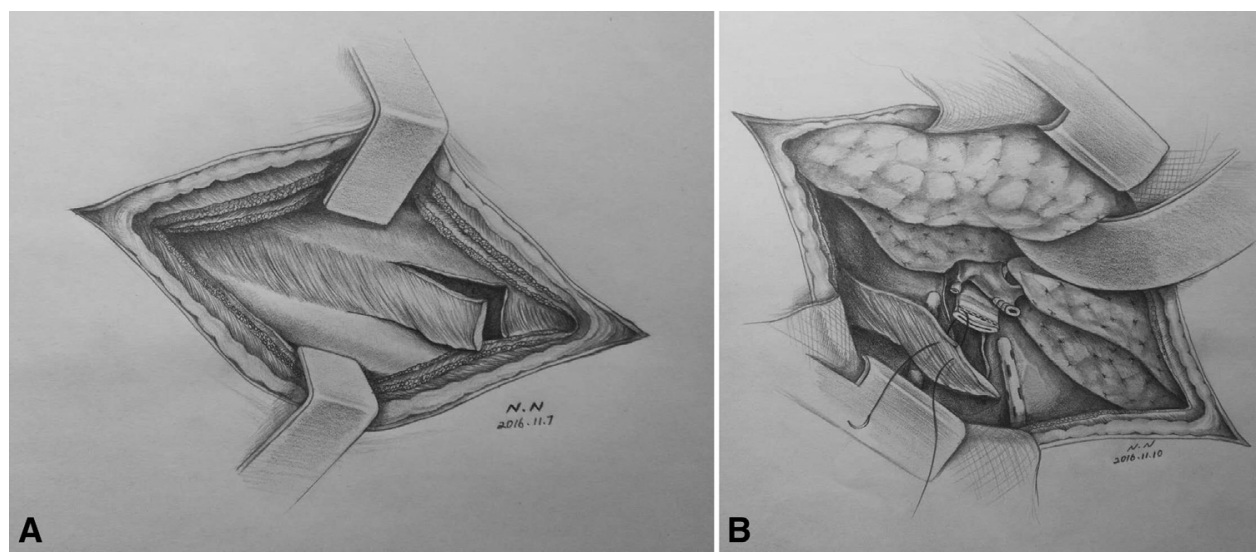

FIGURE E1. The bronchial stump was reinforced with (A), an intercostal muscle flap and (B), 2 chest drains were placed at the site of bronchial stump. 